\title{
Towards Bengali Word Embedding: Corpus Creation, Intrinsic and Extrinsic Evaluations
}

\author{
Md. Rajib Hossain and Mohammed Moshiul Hoque \\ Department of Computer Science \& Engineering, Chittagong University \\ of Engineering and Technology, Chittagong-4349 Bangladesh \\ rajcsecuet@gmail.com, moshiul_240@cuet.ac.bd
}

\begin{abstract}
Distributional word vector representation or word embedding has become an essential ingredient in many natural language processing (NLP) tasks such as machine translation, document classification, information retrieval and question answering. Investigation of embedding model helps to reduce the feature space and improves textual semantic as well as syntactic relations. This paper presents three embedding techniques (such as Word2Vec, GloVe, and FastText) with different hyperparameters implemented on a Bengali corpus consists of 180 million words. The performance of the embedding techniques is evaluated with extrinsic and intrinsic ways. Extrinsic performance evaluated by text classification, which achieved a maximum of $96.48 \%$ accuracy. Intrinsic performance evaluated by word similarity (e.g., semantic, syntactic and relatedness) and analogy tasks. The maximum Pearson $(\hat{r})$ correlation accuracy of $60.66 \%\left(S_{s \hat{r}}\right)$ achieved for semantic similarities and $71.64 \%\left(S_{y \hat{r}}\right)$ for syntactic similarities whereas the relatedness obtained $79.80 \%$ $\left(R_{s \hat{r}}\right)$. The semantic word analogy tasks achieved $44.00 \%$ of accuracy while syntactic word analogy tasks obtained $36.00 \%$.
\end{abstract}

\section{Introduction}

Word embedding is a distributional vector representation of words in which syntactic and semantic interpretations are derived from the enormous amount of unlabeled texts. Recently, the word embedding is considered as a powerful tool due to its many applications in NLP, thus, gained much attention by NLP experts. This is a growing up research issue for well-resourced language like English, where an embedding algorithm generates a model (Devlin et al., 2019). However, it is a very complicated task to adopt an embedding algorithm (of any language) directly for the resourceconstrained languages such as Bengali due to the scarcity of resources. As a result, the low-resource language is trail end in NLP tools development. Bengali is the most popularly communicated language of Bangladesh while second most communicated of the 22 official languages of India which makes Bengali is the $7^{\text {th }}$ most spoken language in the world (Hossain and Hoque, 2019). However, due to the shortage of resources, the development of NLP tools are striving. Bengali speaking people are suffering to access modern NLP tools that might be affected their sustainable use of language technologies. Therefore, Bengali word embedding is an essential prerequisite to developing any Bengali language based NLP tools.

There are two well-known evaluation methods used extensively to evaluate embedding techniques such as extrinsic and intrinsic (Zhelezniak et al., 2019a). Extrinsic evaluation refers to downstream tasks like as machine translation (MT) (Banik et al., 2020), and Part of speech (POS) tagging (Priyadarshi and Saha, 2020). Intrinsic evaluation goals to evaluate the quality of language processing tasks, such as semantic and syntactic word similarity (Pawar and Mago, 2018), Word relatedness (Gladkova and Drozd, 2016), and Word analogy (Schluter, 2018). Unavailability of standard Bengali embedding corpus and inadequacy of resources are antecedents that make such a model generation and evaluation very challenging. Moreover, there is no generalized embedding model available to date for Bengali downstream tasks. Thus, the proposed work introduces a Bengali embedding model generation and evaluation techniques with different hyperparameters settings. Specifically, the key contributions of this work are:

- Acquire raw monolingual Bengali corpora with 180 million words where the unique words are 13 million.

- Construct and annotates the intrinsic and extrinsic evaluation datasets as well as evaluate the annotation purity. 
- Generate ninety embedding models with the combination of three different algorithms (such as Word2Vec, GloVe,and FastText) and variations of model parameters.

- Examine the influence of hyperparameters on the embedding models performance.

As far as we are aware, the proposed work is the first attempt to generate large-scale embedding models evaluates with intrinsic and extrinsic evaluators.

\section{Related Work}

Distributional word vector representation or embedding model generation is a well-established research agenda in NLP domain. There are plenty of research works have been carried out on word embedding in high-resource languages, but it remains as a barrier for low resource languages. The first intrinsic evaluation datasets introduced in RG-65 (Rubenstein and Goodenough, 1965) which contains 65 contextual synonymy pairs. The WordSimilarity-353 introduced by (Finkelstein et al., 2001) and the dataset contains 353 words pair with 13 different subjects. In recent time, three embedding model evaluation datasets have been introduced: SimLex-999 (Hill et al., 2015), SemEval 2017 (Camacho-Collados et al., 2017), and MEN (Bruni et al., 2014). An Italian language embedding model has developed in (Di Gennaro et al., 2020), which achieved $53.74 \%$ overall analogies accuracy for 19,791 texts. Moreover, this work achieved a semantic analogies accuracy of $59.20 \%$ for 8,915 texts and syntactic accuracy of $48.80 \%$ for 10,876 texts. However, this work considered only 3COSADD similarity score and not consider the word relatedness and extrinsic evaluations. Ercan and Yıldiz (2018) devised a Turkish word similarity and relatedness system that produced Turkish embedding dataset derived from English word similarity and relatedness datasets (e. g. WordSimilarity-353, MEN, and SimLex-999). This work achieved spearman score $(\rho)$ of 0.667 for WordSimilarity-353, 0.68 for MEN and 0.67 for SimLex-999 respectively. The developed embedding model was not evaluated with extrinsic evaluation (Chiu et al., 2016).

Although most of the current works on embedding model, resource creation and evaluations conducted for the high-resource languages (e.g., English, Germany, and French) there are few compre- hensive research conducted on low resource languages such as Assamese, Gujarati, Hindi, Kannada, and so on (Kumar et al., 2020) and Turkish (Ercan and Y1ldız, 2018). Kumar et al. (2020) introduced the pre-trained word embedding models for Indian languages. The proposed system generates 14 Indian local language embeddings with 8 different approaches, including 436 models. Embedding models are evaluated by extrinsic evaluators and archived more than $90.00 \%$ accuracy for UPOS, and XPOS tagging using universal dependency treebank datasets (Nivre et al., 2016). The NER tagging accuracy about $95.00 \%$ with FastText embedding. Kumar et al. (2020) aimed to solve 14 Indian languages NLP problems, but the generated models are not considered intrinsic evaluations. To best of our knowledge, only single research was conducted concerning Bengali word embedding using Word2Vec (Sadman et al., 2019). However, this work considered only intrinsic evaluations with a self-build dataset. Our approach considered ninety embedding models based on GloVe, FastText and Word2 Vec models and measured the performance using intrinsic and extrinsic evaluators.

\section{Methodology}

The principal aim of our research is to investigate the affect of intrinsic and extrinsic evaluations on Bengali word embedding models. Thus, the proposed scheme comprises of three main parts: corpus creation, word embedding model development, and evaluation. Figure 1 illustrates the abstract view of our work.

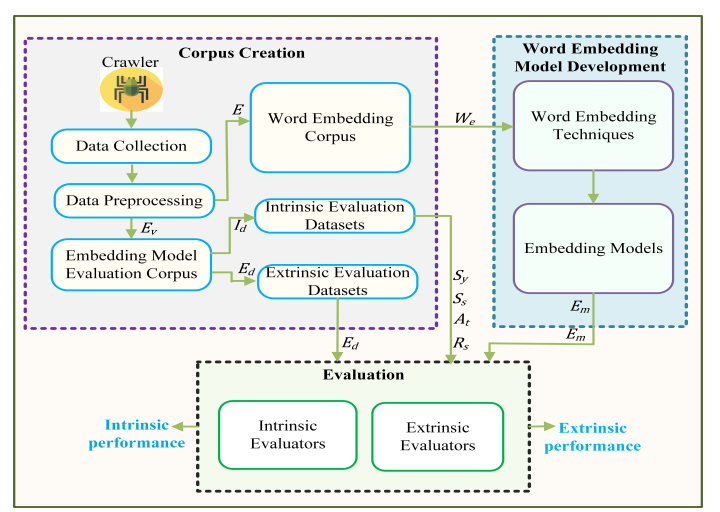

Figure 1: Abstract process of evaluations for Bengali word embedding.

\subsection{Corpus Creation}

We collected Bengali texts from various online sources and distributed these into two sets: word 
embedding corpus $(E)$ and embedding model evaluation corpus $\left(E_{v}\right)$. We used a Python crawler to crawl data. We collected 910,720 Bengali text files over a twenty-four month period (September 10, 2018, to September 11, 2020) which are forwarded to data preprocessing step. Initially, the non-Bengali alphabets and digits are removed from the text files. In the next, preprocessing step removed HTML tags, hashtags, URLs, punctuation and white spaces. Finally, the duplicate texts are deleted from the archive. The preprocessing step produced of 882,352 usable text, and removed 28,368 blank size text documents from the initial dataset due to various preprocessing operations. These usable preprocessed data are randomly distributed into two sets; one set for embedding model evaluation (100, 000 texts) and another set for word embedding corpus (782, 352 texts). The embedding corpus $\left(W_{e}\right)$ (i.e., total 180, 081, 093 words) is fed to the embedding techniques.

Embedding model evaluation corpus $\left(E_{v}\right)$ is a combination of intrinsic $\left(I_{d}\right)$ and extrinsic datasets $\left(E_{d}\right)$. In order to perform an extrinsic evaluation, out of 100,000 text documents, a total of 60,000 documents are randomly selected. This dataset was labelled manually followed by majority voting to assign the suitable label. Two linguistic experts are assigned to annotated each data into one of the six pre-defined categories such as Accident $\left(A_{t}\right)$, Crime $\left(C_{e}\right)$, Entertainment $\left(E_{t}\right)$, Health $\left(H_{h}\right)$, Politics $\left(P_{s}\right)$ and, Sports $\left(S_{p}\right)$. Among 60,000 text documents, both experts have been agreed upon 54,858 text labels. The developed corpus $\left(E_{d}\right)$ achieved a Kappa score $(K)$ is $78.53 \%$, which indicates a reasonable agreement between annotators for downstream task. To perform intrinsic evaluations of the embedding models four different sub datasets are used for conducting four measures: semantic word similarity $\left(S_{s}\right)$, syntactic word similarity $\left(S_{y}\right)$, relatedness $\left(R_{r}\right)$, and analogy tasks $\left(A_{t}\right)$. Intrinsic datasets and corresponding kappa score are shown in Table 1 . Here, $S_{s}, S_{y}$ and $R_{s}$

\begin{tabular}{lll}
\hline Datasets & No. of samples & Kappa score (\%) \\
\hline$S_{s}$ & 100 & 71.55 \\
$S_{y}$ & 100 & 65.30 \\
$R_{s}$ & 100 & 75.13 \\
$A_{t}$ & 100 & 56.90 \\
\hline
\end{tabular}

Table 1: Embedding model datasets and kappa score.

datasets are substantial agreement where as $A_{t}$ is moderate agreement.

\subsection{Word Embedding Model Development}

We consider three well-known embedding techniques for Bengali corpus including Word2Vec, GloVe, and FastText. To realize the effect of hyperparameters, we have considered embedding dimension (size), minimum word frequency count (min_count), contextual windows size (window) and number of iteration (epoch) for each of the embedding technique.

\section{Word2Vec (skip-gram and CBOW):} Word2Vec (Mikolov et al., 2013a) technique takes $W_{e}$ as the input and produce the embedding model $E_{m}$ using gensim library (Řehưřek and Sojka, 2010). Two versions of Word2 Vec pre-trained models such as Skip-gram and continuous bag of words (CBOW) are used with similar hyperparameters for tuning: size : $\{50,100,150,200,250,300\}$, window : $\{5,10,15\}$, min_count : $\{2\}$ and epochs : 30 respectively. There are 36 embedding models (e.g., 18 for CBOW and 18 for skip-gram) generated for $W_{e}$ using Word2Vec technique.

GloVe: GloVe (Pennington et al., 2014) technique generated 18 embedding models $\left(E_{m}\right)$ for the embedding corpus $W_{e}$. Different hyperparameters are used to optimize the model such as size : $\{50,100,150,200,250,300\}$, min_count : $\{2\}, X_{-} M A X: 95$, epochs : 30 and window : $\{5,10,15\}$ respectively. The remaining hyperparameters remains the same as default settings. Finally, the eighteen embedding models $\left(E_{m}\right)$ are evaluated by the evaluators.

FastText (skip-gram and CBOW): FastText embedding technique (Bojanowski et al., 2017), takes $W_{e}$ as input and generates a embedding model $\left(E_{m}\right)$ as output using gensim library (Ǩehưřek and Sojka, 2010). Different values of hyperparameters are used to achieve optimize performance such as size : $\{50,100,150,200,250,300\}$, window : $\{5,10,15\}$, min_count : $\{2\}$ and epochs : 30 respectively. Our approach produced of 36 models (e.g., 18 for FastText-skip-gram and 18 for FastText-CBOW) that are evaluated using evaluators.

\section{Results and Discussion}

Intrinsic evaluations are performed for a total of ninenty (e.g., Word2Vec=36, GloVe $=18$ and Fast- 


\begin{tabular}{|c|c|c|c|c|c|c|c|c|}
\hline \multirow[b]{2}{*}{ Model } & \multirow[b]{2}{*}{ size } & \multirow[b]{2}{*}{ window } & \multicolumn{2}{|c|}{ Semantic (\%) } & \multicolumn{2}{|c|}{ Syntactic (\%) } & \multicolumn{2}{|c|}{ Relatedness (\%) } \\
\hline & & & $S_{s \hat{\rho}}$ & $S_{s \hat{r}}$ & $S_{y \hat{\rho}}$ & $S_{y \hat{r}}$ & $R_{s \hat{\rho}}$ & $R_{y \hat{r}}$ \\
\hline \multirow{3}{*}{ GloVe } & 300 & 15 & 60.02 & 60.66 & 69.54 & 70.62 & 79.20 & 79.72 \\
\hline & 250 & 10 & 56.33 & 57.77 & 70.41 & 70.66 & 79.22 & 79.80 \\
\hline & 250 & 5 & 56.93 & 57.90 & 69.87 & 71.64 & 79.20 & 78.33 \\
\hline \multirow[t]{2}{*}{ FastText (SG) } & 250 & 10 & 53.64 & 53.38 & 39.47 & 38.31 & 68.28 & 67.54 \\
\hline & 150 & 10 & 56.78 & 55.18 & 37.78 & 36.03 & 66.63 & 65.84 \\
\hline Word2Vec (SG) & 250 & 5 & 44.31 & 45.10 & 31.78 & 30.51 & 56.43 & 57.41 \\
\hline $\begin{array}{l}\text { FastText (Grave } \\
\text { et al., 2018) }\end{array}$ & 300 & - & 49.41 & 49.78 & 5.93 & -1.55 & 47.23 & 43.91 \\
\hline
\end{tabular}

Table 2: Performance of embedding models concerning semantic, syntactic and relatedness word similarity.

Text=36) embedding models. Among these, the results of best four embedding models presented for extrinsic and intrinsic evaluators.

\subsection{Intrinsic evaluation results}

The word similarity (semantic and syntactic) score is calculated by Cosine similarity $(C)$. The model performance can be calculated from the spearman $(\hat{\rho})$ and pearson $(\hat{r})$ correlations (Zhelezniak et al., 2019b). The well-known word analogy solver, 3COSADD (Mikolov et al., 2013b) is used to solve the analogy tasks. Three similarity measures are used to evaluate word similarity and analogy tasks analysis. In order to maintain consistency, we performed training for all models with our developed corpus.

Word similarity: Table 2 shows the intrinsic evaluations performance of the embedding models. Annotators word similarity rates are range from $0-10$ whereas the cosine similarity score normalized by ten times. All values in Table 2 are normalized by hundreds times. Maximum semantic correlation values are $S_{s \hat{\rho}}=60.02 \%$ and $S_{s \hat{r}}=60.66 \%$ for GloVe (size $=300$ and window $=15$ ) technique. Highest syntactic correlation is $S_{y \hat{\rho}}=70.41 \%$ for GloVe (size $=250$ and window $=10)$ where as $S_{y \hat{r}}=71.64 \%$ for GloVe $($ size $=250$ and window $=5)$ techniques. The $R_{s}$ highest correlations, $R_{s \hat{\rho}=79.22 \%}$ and $R_{y \hat{r}=79.80 \%}$ have been achieved using GloVe $($ size $=250$ and window $=10)$ technique. There are eight semantic words pair are not able to process by $E_{m}$ where as four syntactic words pair are not able to process by $E_{m}$ of all techniques. Relatedness words pair are fully processed by all embedding techniques.
Word analogy results: The semantic analogy results are shown in Table 3, while Table 4 denotes the syntactic analogy tasks performance based on our corpus. Due to unavailability of Bengali semantic and syntactic analogy datasets, we have been developed $A_{t}$ datasets were 50 analogy words used for semantic and another fifty used to perform syntactic analogy tasks. GloVe (size $=300$ and window $=15)$ technique has achieved maximum accuracy of $38.00 \%$ (Add) and $44.00 \%$ (Mull) for semantic analogy tasks. Minimum semantic analogy tasks accuracy is obtained by FastText (Grave et al., 2018) embedding model. The maximum

\begin{tabular}{lcccc}
\hline \multicolumn{5}{c}{ Semantic analogy tasks accuracy (\%) } \\
\hline Model & size & window & Add & Mull \\
\hline GloVe & $\mathbf{3 0 0}$ & $\mathbf{1 5}$ & $\mathbf{3 8 . 0 0}$ & $\mathbf{4 4 . 0 0}$ \\
FastText (SG) & 250 & 10 & 30.00 & 34.00 \\
Word2Vec(SG) & 250 & 5 & 26.00 & 30.00 \\
FastText & 300 & - & 20.00 & 26.00 \\
(Grave et al., & & & & \\
2018) & & & & \\
\hline
\end{tabular}

Table 3: Analogy tasks performance summary for semantic datasets.

syntactic analogy tasks accuracy are $30.00 \%$ (Add) and $36.00 \%$ achieved by GloVe (size $=300$ and window $=15) E_{m}$, while $20.00 \%$ (Add) and $24.00 \%$ (Mull) from FastText (Grave et al., 2018) embedding model.

\subsection{Extrinsic evaluation results}

The $E_{d}$ is a Bengali text classification dataset which partitioned into the three sets: training $(39,079)$, validation $(6,000)$ and testing $(9,779)$. The text classifier model trained with a multi-kernel CNN architecture (Kim, 2014). The performance of the text classifier model assesses with extrinsic 


\begin{tabular}{lcccl}
\hline \multicolumn{5}{c}{ Syntactic analogy tasks accuracy (\%) } \\
\hline Model & size & window & Add & Mull \\
\hline GloVe & $\mathbf{3 0 0}$ & $\mathbf{1 5}$ & $\mathbf{3 0 . 0 0}$ & $\mathbf{3 6 . 0 0}$ \\
FastText (SG) & 250 & 10 & 26.00 & 28.00 \\
Word2Vec(SG) & 250 & 5 & 20.00 & 26.00 \\
FastText & 300 & - & 20.00 & 24.00 \\
(Grave et al., & & & & \\
2018) & & & & \\
\hline
\end{tabular}

Table 4: Analogy tasks performance for syntactic datasets.

evaluators including accuracy $(A)$, micro average F1-score, average precision $\left(A_{p}\right)$, average recall $\left(A_{r}\right)$ and confusion matrix (CM) (Wu et al., 2020). The evaluators evaluated the embedding models $\left(E_{m}\right)$ downstream task (e.g., text classification) performance (in Tables 5 and 6). Table 5 shows the summary of text classification performance.

\begin{tabular}{llll}
\hline Models & size/window & F1-score(\%) & $\mathrm{A}(\%)$ \\
\hline Word2Vec & $250 / 10$ & 93.43 & 93.87 \\
GloVe & $\mathbf{2 0 0} / \mathbf{1 0}$ & $\mathbf{9 6 . 0 3}$ & $\mathbf{9 6 . 4 8}$ \\
FastText & $200 / 15$ & 95.57 & 95.71 \\
\hline
\end{tabular}

Table 5: Extrinsic evaluation for text classification.

The GloVe model achieved the highest accuracy of $96.48 \%$. For clarity, we presented only the results of best four embedding models out of ninety models. Table 6 depicts the confusion matrix of GloVe model $($ size $=200$ and window $=10)$ for text classification performance. The maximum correctly predicted class is Politics and incorrectly predicted class is Crime. The highest misclassification occurred for Crime and Accident pair.

\begin{tabular}{ccccccc}
\hline $\mathrm{CM}$ & $A_{t}$ & $C_{e}$ & $E_{t}$ & $H_{h}$ & $P_{s}$ & $S_{p}$ \\
\hline$A_{t}$ & 1636 & 43 & 1 & 3 & 3 & 2 \\
$C_{e}$ & 62 & 1468 & 2 & 10 & 27 & 3 \\
$E_{t}$ & 1 & 4 & 1603 & 12 & 8 & 16 \\
$H_{h}$ & 1 & 5 & 16 & 1593 & 12 & 9 \\
$P_{s}$ & 3 & 15 & 3 & 11 & 1570 & 6 \\
$S_{p}$ & 1 & 6 & 43 & 4 & 12 & 1565 \\
\hline
\end{tabular}

Table 6: Confusion matrix of text classification task.

Figure 2 shows few example scores for semantic, syntactic and relatedness words pair score obtained from GloVe model and human annotators. GloVe and FastText (SG) models accuracy are considerable for semantic and relatedness similarities. In the case of extrinsic evaluations, the performance

\begin{tabular}{|c|c|c|c|c|}
\hline \multirow{3}{*}{$\begin{array}{l}\text { Semantic } \\
\text { Words pair }\end{array}$} & Word $_{1}$ & Word $_{2}$ & \multirow{2}{*}{$\begin{array}{l}\text { Annotators } \\
\text { score ( Avg.) }\end{array}$} & \multirow{2}{*}{$\begin{array}{c}\text { Cosine } \\
\text { Similarity (C) }\end{array}$} \\
\hline & \multirow{2}{*}{$\begin{array}{l}\text { অপরাধ } \\
\text { Aparādha } \\
\text { Crime }\end{array}$} & \multirow{2}{*}{\begin{tabular}{|l|} 
পাপ \\
pāpa \\
Sin \\
\end{tabular}} & & \\
\hline & & & 4.00 & 3.10 \\
\hline $\begin{array}{l}\text { Semantic } \\
\text { Words pair }\end{array}$ & \begin{tabular}{|l} 
জল \\
Jala \\
Water \\
\end{tabular} & $\begin{array}{l}\text { পানি } \\
\text { Pāni } \\
\text { Water } \\
\end{array}$ & 8.80 & 6.69 \\
\hline $\begin{array}{l}\text { Relatedness } \\
\text { Words pair }\end{array}$ & \begin{tabular}{|l} 
স্কুল \\
Skula \\
School \\
\end{tabular} & $\begin{array}{l}\text { कলেজ } \\
\text { kalēja } \\
\text { College } \\
\end{array}$ & 8.50 & 7.62 \\
\hline $\begin{array}{l}\text { Relatedness } \\
\text { Words pair }\end{array}$ & \begin{tabular}{|l|} 
শার্ট \\
S̄arța \\
Shirt \\
\end{tabular} & $\begin{array}{l}\text { প্যান্ট } \\
\text { Pyānța } \\
\text { Pants }\end{array}$ & 8.70 & 8.32 \\
\hline $\begin{array}{l}\text { Syntactic } \\
\text { Words pair }\end{array}$ & $\begin{array}{l}\text { শিক্ষক } \\
\text { Śikșaka } \\
\text { Teacher }\end{array}$ & $\begin{array}{l}\text { শিক্ষকরা } \\
\text { Śikșakarā } \\
\text { Pants }\end{array}$ & 7.3 & 6.60 \\
\hline $\begin{array}{l}\text { Syntactic } \\
\text { Words pair }\end{array}$ & $\begin{array}{l}\text { পর্বত } \\
\text { Parbata } \\
\text { Mountain }\end{array}$ & $\begin{array}{l}\text { পর্বতমালা } \\
\text { Parbatamāāā } \\
\text { Mountains }\end{array}$ & 4.30 & 3.25 \\
\hline
\end{tabular}

Figure 2: Word pair similarity scores, the Cosine similarity score is normalized by 10 times and annotators score is ranging between 1 to 10 .

of GloVe and FastText embedding models are significant for the text classification task.

\section{Conclusion and Future Work}

In this work, we have been generated about ninety embedding models for the Bengali language. These models have developed using the combinations of three embedding techniques (such as GloVe, Word2Vec, and FastText) and various hyperparameters. All models have evaluated by extrinsic and intrinsic evaluators on our developed corpus. The performance of an embedding model significantly depends on the hyperparameters, corpus and nature of the model. Although GloVe model performed better than Word2 Vec and FastText, there is no generalized embedding model for intrinsic and extrinsic NLP tasks. The embedding models are highly corpus oriented, and hyperparameters also vary from one task to another. In the future, the existing Bengali corpus can be extended for embedding model generation to alleviate the outof-vocabularies problems. The context-dependent feature represents technique (such as BERT, EIMo and XLNet) will be investigated to find suitable embedding technique for Bengali. In addition to that, more analogy tasks can be considered to assess the performance of different embedding models with various intrinsic and extrinsic evaluators.

\section{References}

Debajyoty Banik, Asif Ekbal, and Pushpak Bhattacharyya. 2020. Statistical machine translation based on weighted syntax-semantics. Sädhanā, 45:1-12.

Piotr Bojanowski, Edouard Grave, Armand Joulin, and 
Tomas Mikolov. 2017. Enriching word vectors with subword information. Transactions of the Association for Computational Linguistics, 5:135-146.

Elia Bruni, Nam Khanh Tran, and Marco Baroni. 2014. Multimodal distributional semantics. Journal of Artificial Intelligence Research, 49(1):1-47.

Jose Camacho-Collados, Mohammad Taher Pilehvar, Nigel Collier, and Roberto Navigli. 2017. SemEval2017 task 2: Multilingual and cross-lingual semantic word similarity. In Proceedings of the 11th International Workshop on Semantic Evaluation (SemEval2017), pages 15-26, Vancouver, Canada. Association for Computational Linguistics.

Billy Chiu, Anna Korhonen, and Sampo Pyysalo. 2016. Intrinsic evaluation of word vectors fails to predict extrinsic performance. In Proceedings of the 1st Workshop on Evaluating Vector-Space Representations for NLP, pages 1-6, Berlin, Germany. Association for Computational Linguistics.

Jacob Devlin, Ming-Wei Chang, Kenton Lee, and Kristina Toutanova. 2019. BERT: Pre-training of deep bidirectional transformers for language understanding. In Proceedings of the 2019 Conference of the North American Chapter of the Association for Computational Linguistics: Human Language Technologies, Volume 1 (Long and Short Papers), pages 4171-4186, Minneapolis, Minnesota. Association for Computational Linguistics.

Giovanni Di Gennaro, Amedeo Buonanno, Antonio Di Girolamo, Armando Ospedale, Francesco A. N. Palmieri, and Gianfranco Fedele. 2020. An analysis of word2vec for the italian language. Smart Innovation, Systems and Technologies, pages 137-146.

Gökhan Ercan and Olcay Taner Yildız. 2018. AnlamVer: Semantic model evaluation dataset for Turkish - word similarity and relatedness. In Proceedings of the 27th International Conference on Computational Linguistics, pages 3819-3836, Santa Fe, New Mexico, USA. Association for Computational Linguistics.

Lev Finkelstein, Evgeniy Gabrilovich, Yossi Matias, Ehud Rivlin, Zach Solan, Gadi Wolfman, and Eytan Ruppin. 2001. Placing search in context: The concept revisited. In Proceedings of the 10th International Conference on World Wide Web, page 406-414, New York, NY, USA. Association for Computing Machinery.

Anna Gladkova and Aleksandr Drozd. 2016. Intrinsic evaluations of word embeddings: What can we do better? In Proceedings of the 1st Workshop on Evaluating Vector-Space Representations for NLP, pages 36-42, Berlin, Germany. Association for Computational Linguistics.

Edouard Grave, Piotr Bojanowski, Prakhar Gupta, Armand Joulin, and Tomas Mikolov. 2018. Learning word vectors for 157 languages. In Proceedings of the Eleventh International Conference on Language Resources and Evaluation (LREC-2018), Miyazaki, Japan. European Languages Resources Association (ELRA).

Felix Hill, Roi Reichart, and Anna Korhonen. 2015. SimLex-999: Evaluating semantic models with (genuine) similarity estimation. Computational Linguistics, 41(4):665-695.

Md. Rajib Hossain and Mohammed Moshiul Hoque. 2019. Automatic bengali document categorization based on deep convolution nets. In Emerging Research in Computing, Information, Communication and Applications, pages 513-525, Singapore. Springer Singapore.

Yoon Kim. 2014. Convolutional neural networks for sentence classification. In Proceedings of the 2014 Conference on Empirical Methods in Natural Language Processing (EMNLP), pages 1746-1751, Doha, Qatar. Association for Computational Linguistics.

Saurav Kumar, Saunack Kumar, Diptesh Kanojia, and Pushpak Bhattacharyya. 2020. "a passage to India": Pre-trained word embeddings for Indian languages. In Proceedings of the 1st Joint Workshop on Spoken Language Technologies for Under-resourced languages (SLTU) and Collaboration and Computing for Under-Resourced Languages (CCURL), pages 352-357, Marseille, France. European Language Resources association.

Tomas Mikolov, Kai Chen, Greg Corrado, and Jeffrey Dean. 2013a. Efficient estimation of word representations in vector space. CoRR, abs/1301.3781.

Tomas Mikolov, Wen-tau Yih, and Geoffrey Zweig. 2013b. Linguistic regularities in continuous space word representations. In Proceedings of the 2013 Conference of the North American Chapter of the Association for Computational Linguistics: Human Language Technologies, pages 746-751, Atlanta, Georgia. Association for Computational Linguistics.

Joakim Nivre, Marie-Catherine de Marneffe, Filip Ginter, Yoav Goldberg, Jan Hajič, Christopher D. Manning, Ryan McDonald, Slav Petrov, Sampo Pyysalo, Natalia Silveira, Reut Tsarfaty, and Daniel Zeman. 2016. Universal dependencies v1: A multilingual treebank collection. In Proceedings of the Tenth International Conference on Language Resources and Evaluation (LREC 2016), pages 1659-1666, Portorož, Slovenia. European Language Resources Association (ELRA).

Atish Pawar and Vijay Mago. 2018. Calculating the similarity between words and sentences using a lexical database and corpus statistics. CoRR, abs/1802.05667.

Jeffrey Pennington, Richard Socher, and Christopher Manning. 2014. Glove: Global vectors for word representation. In Proceedings of the 2014 Conference 
on Empirical Methods in Natural Language Processing (EMNLP), pages 1532-1543, Doha, Qatar. Association for Computational Linguistics.

Ankur Priyadarshi and Sujan Kumar Saha. 2020. Towards the first maithili part of speech tagger: Resource creation and system development. Computer Speech Language, 62:101054.

Herbert Rubenstein and John B. Goodenough. 1965. Contextual correlates of synonymy. Commun. ACM, 8(10):627-633.

Nafiz Sadman, Akib Sadmanee, Md. Iftekhar Tanveer, Md. Ashraful Amin, and Amin Ahsan Ali. 2019. Intrinsic evaluation of bangla word embeddings. In 2019 International Conference on Bangla Speech and Language Processing (ICBSLP), pages 1-5. IEEE.

Natalie Schluter. 2018. The word analogy testing caveat. In Proceedings of the 2018 Conference of the North American Chapter of the Association for Computational Linguistics: Human Language Technologies, Volume 2 (Short Papers), pages 242-246, New Orleans, Louisiana. Association for Computational Linguistics.

Di Wu, Mengtian Zhang, Chao Shen, Zhuyun Huang, and Mingxing Gu. 2020. Btm and glove similarity linear fusion-based short text clustering algorithm for microblog hot topic discovery. volume 8, pages 32215-32225. IEEE Access.

Vitalii Zhelezniak, Aleksandar Savkov, April Shen, and Nils Hammerla. 2019a. Correlation coefficients and semantic textual similarity. In Proceedings of the 2019 Conference of the North American Chapter of the Association for Computational Linguistics: $\mathrm{Hu}$ man Language Technologies, Volume 1 (Long and Short Papers), pages 951-962, Minneapolis, Minnesota. Association for Computational Linguistics.

Vitalii Zhelezniak, Aleksandar Savkov, April Shen, and Nils Hammerla. 2019b. Correlation coefficients and semantic textual similarity. In Proceedings of the 2019 Conference of the North American Chapter of the Association for Computational Linguistics: $\mathrm{Hu}$ man Language Technologies, Volume 1 (Long and Short Papers), pages 951-962, Minneapolis, Minnesota. Association for Computational Linguistics.

Radim Řehůřek and Petr Sojka. 2010. Software framework for topic modelling with large corpora. In Proceedings of LREC 2010 workshop New Challenges for NLP Frameworks, pages 46-50, Valletta, Malta. University of Malta. 